\title{
Energy Dependence of a Vortex Line Length Near a Zigzag of Pinning Centers
}

\author{
Mauro M. Doria and Antonio R. de C. Romaguera \\ Instituto de Física, Universidade Federal do Rio de Janeiro \\ C.P. 68528, 21941-972, Rio de Janeiro RJ, Brazil
}

Received on 12 July, 2004

\begin{abstract}
A vortex line, shaped by a zigzag of pinning centers, is described here through a three-dimensional unit cell containing two pinning centers positioned symmetrically with respect to its center. The unit cell is a cube of side $L=12 \xi$, the pinning centers are insulating spheres of radius $R$, taken within the range $0.2 \xi$ to $3.0 \xi$, $\xi$ being the coherence length. We calculate the free energy density of these systems in the framework of the Ginzburg-Landau theory.
\end{abstract}

\section{Introduction}

Superconductors have many kinds of imperfections, that is, internal regions where Cooper pairs either don't exist at all or exist purely as a fluctuation effect. Thus the macroscopic wave function, describing the collective state, vanishes abruptly or asymptotically inside such regions. In presence of an external magnetic field vortices arise inside the superconductor and are strongly attracted to such imperfections, also called pinning centers. For this reason pinning centers have been extensively studied in the past in many ways, including artificially made ones, such as columnar defects [1], antidots [2,3] and micro holes [4]. They are interesting because they bring clear-cut questions about the interaction between vortices and pinning centers [5,6], such as how local misalignment really occurs inside the superconductor. Vortices lines should be aligned to the applied field but the presence of strong attraction to a pinning center can change this locally. As a result of competing energetic demands new interesting phenomena can take place in vortex Physics, such as the one considered here. A vortex line in the absence of pinning centers is aligned along the magnetic induction direction, hereafter called z-axis. The presence of a zigzag of pinning centers forces the vortex line to bend and acquire this shape, resulting into local misalignment, though it remains oriented along the magnetic induction.

Pinning forces act on the vortex core whose radius is given by the coherence length $\xi$. The interaction of pinning centers with vortices has been studied using several approaches [7]. From the point of view of the GinzburgLandau theory, pinning may be caused by spatial fluctuations of the critical temperature [8], $T_{c}(\vec{x})$, or of the mean free-path [9] that changes the coefficient in front of the gradient term, $\xi(\vec{x})^{2}\left|\left(\vec{\nabla}-\frac{2 \pi i}{\Phi_{0}} \vec{A}\right) \Delta\right|^{2}$. The interaction between a vortex line and a pinning center has been considered by many authors in the context of the Ginzburg-Landau theory $[9,10]$. The number of vortex that can be trapped by a defect is an interesting problem. In case of a columnar defect the saturation number has been determined long ago by Mkrtchyan and Shmidt [11]: $n_{s}=\lambda / 2 \xi$, though this formula has to change near the upper critical field [12]. Recently the saturation number has been discussed for three dimensional cavities [13].

The system under investigation here consists of a vortex line trapped by a zigzag of very large pinning centers, namely, spherical insulating cavities, with radius taken to vary from $0.2 \xi$ to $3.0 \xi$. For these pinning centers the boundary-value problem has to be taken into account, as usually treated since the de Gennes boundary condition [14] must be satisfied at the cavity surface. We chose to describe this system through a unit cell with two cavities inside, that rotate freely around its center, producing for each angle a distinct zigzag arrangement. In this paper we analyze numerically the angular dependence of the Helmholtz free energy $\mathcal{F}_{c}(\theta, R)$ and propose an expression for it.

This paper is organized as follows. In section 2 we present the model for a 3-D superconducting media with the cavities. In section 3 , we discuss out theoretical approach. In sections 4 and 5 we show the results obtained through numerical simulations. In section 6 we summarize the main results of the work.

\section{The model}

The system studied here consists of a cubic unit cell with size $L$ equal to $12 \xi$, and two insulating cavities of radius $R$, as specified by the Fig. 1 . The line segment joining the cavities makes an angle $\theta$ ranging from $0^{\circ}$ to $180^{\circ}$, taken here in increments of $3^{\circ}$. Notice that because of symmetry $\theta$ varying from $0^{\circ}$ to $90^{\circ}$ is sufficient to obtain all possible configurations.

Figure 1 shows the plane of rotation (plane y) and parameters associated to the unit cell. While $\theta$ and $R$ are freely changed, $D$, the distance between the center of the cavities, is fixed and equal to $L / 2$. Thus the present model features a distance between the two cavities independent of the cavity 
radius.

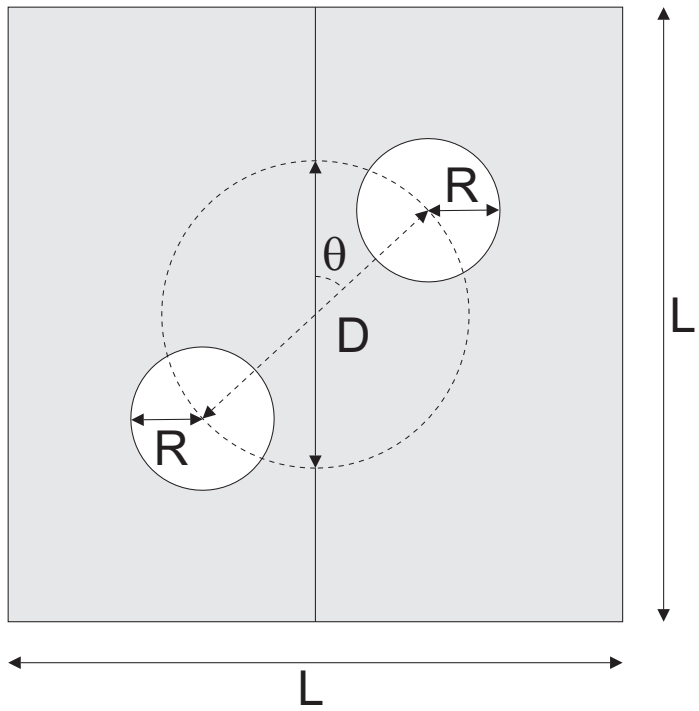

Figure 1. The plane of rotation of the cavities inside the unit cell. The distance $D$ between the two cavities is equal to $L / 2$. The superconductor fills the remain of the unit cell, shown here as a gray region.

Our numerical treatment demands a mesh grid to describe the unit cell. The length of the cube side is $12 \xi$, and we choose the number of mesh points along a given direction, $P$, to be 19 . This choice implies that distance between two consecutive mesh points, $a$, be equal to $2 \xi / 3$. The value of $a$ must be smaller than the coherence length $\xi$, which is the minimum physical scale of the GinzburgLandau theory. The number of grid points inside the cavity should be large enough to describe it. This number is obtained from the ratio between the two volumes, of the cavity and of the cube. Since the entire cube has $P^{3}$ points, the number of grid points inside the cavity is $\frac{4}{3} \pi\left(\frac{R}{a}\right)^{3}\left(1+\frac{a}{L}\right)^{3}$. For instance, a cavity of radius $R=1.0 \xi$ has approximately 16 points, whereas the $R=2.0 \xi$ sphere has 8 times more points. Obviously in the limit of a cavity with radius smaller thant the mesh distance, that is $R<a=2 / 3 \xi$, there will be just one point in the mesh describing the cavity. In this case the order parameter does not vanish inside the defect but just undergoes a drop on its value.

\section{Theoretical approach}

We start our considerations for the energy density functional of the Ginzburg-Landau theory [15], expressing it in units of the critical field energy density [13], $H_{c}^{2} / 4 \pi$. The periodicity of the problem requires a search of the free energy minimum for a fixed integer, the number of vortices inside the unit cell.

This integer also fixes the magnetic induction $\vec{B}(\vec{x})$, which is the average of the local field taken over the unit cell volume,

$$
\vec{B}=\frac{1}{v} \int_{v} \vec{h} d^{3} r
$$

The magnetic induction is completely determined by the vorticity of the system because the overall current circulation vanishes inside the unit cell. The relationship between the vorticity of the system $\vec{\nu} \phi_{0}$ and the magnetic induction $\vec{B}(\vec{x})$ in reduced units is,

$$
\vec{B}(\vec{x})=2 \pi \kappa\left(\frac{\xi}{L}\right)^{2} \vec{\nu}
$$

where $\vec{\nu}=n_{x} \hat{x}+n_{y} \hat{y}+n_{z} \hat{z}$ is the vorticity in an arbitrary direction. In the present paper we consider $\vec{\nu}=\hat{z}$ and present some results concerning the $\vec{\nu}=2 \hat{z}$ case. The parameter $\kappa=\lambda / \xi$ is the dimensionless Ginzburg-Landau parameter and $\lambda$ is the penetration depth. Here we consider the no magnetic shielding limit. The field penetrates in the superconductor with no Meissner-Ochsenfeld effect. In this regime $\vec{h}(\vec{x})=\nabla \times \vec{A}(\vec{x})=\vec{B}$. This situation can be viewed as a large $\kappa$ limit. In reduced units the free energy density is normalized by the critical field density, $H_{c}^{2} / 4 \pi$, and the order parameter density is dimensionless, varying between 0 and 1.

$$
\begin{aligned}
\mathcal{F}_{c} & =\int \frac{d v}{V} \tau(\vec{x})\left[\xi^{2}\left|\left(\vec{\nabla}-\frac{2 \pi i}{\Phi_{0}} \vec{A}\right) \Delta\right|^{2}-|\Delta|^{2}\right] \\
& +\frac{1}{2}|\Delta|^{4}
\end{aligned}
$$

The function $\tau(\vec{x})$ is a step-like function used to specify the cavities in this approach [13]. Explicitly we have $\tau(\vec{x})=\tau_{1}(\vec{x}) \tau_{2}(\vec{x})$ and

$$
\tau_{i}(\vec{x})=1-\frac{2}{1+e^{\left(\left|\vec{x}-\vec{x}_{i}\right| / R\right)^{N}}}
$$

where $\tau_{i}$ is equal to 0 inside and 1 outside the $i$ th cavity. The above explicit representation of the $\tau$ function is necessary for computational reasons and for accuracy we take that $N=8$. In the limit $N \rightarrow \infty$, the function $\tau$ tends to the well-known Heaviside function, $\tau(\vec{x})=$ $\Theta\left(\frac{\left|\vec{x}-\vec{x}_{1}\right|}{R}-1\right) \Theta\left(\frac{\left|\vec{x}-\vec{x}_{2}\right|}{R}-1\right)$.

Since we are in the no shielding limit, the vector potential $\vec{A}(\vec{x})$ is determined from Eqs. 1, 2 and the condition of magnetic flux quantization inside the unit cell. The vector potential does not participate in the minimization process of the free energy density that only takes into account the real and imaginary parts of the order parameter. The free energy density contains two terms. The first term is the condensation energy density, $-\tau(\vec{x})|\Delta|^{2}+\frac{1}{2}|\Delta|^{4}$, which in case of no vortices $\left(|\Delta|^{2}=1\right)$ and no cavity $(\tau=1)$ has the value -0.5 . The presence of a cavity raises the energy since inside it the density vanishes $\left(|\Delta|^{2}=0\right)$. And the second term, the kinetic energy density, $\tau(\vec{x}) \xi^{2}\left|\left(\vec{\nabla}-\frac{2 \pi i}{\Phi_{0}} \vec{A}\right) \Delta\right|^{2}$. Notice that there is kinetic energy in case of no vortices but with a cavity. At the insulating-superconducting interface $\tau$ changes from 1 to 0 and this causes a bending of the order parameter, which has some kinetic energy cost. 


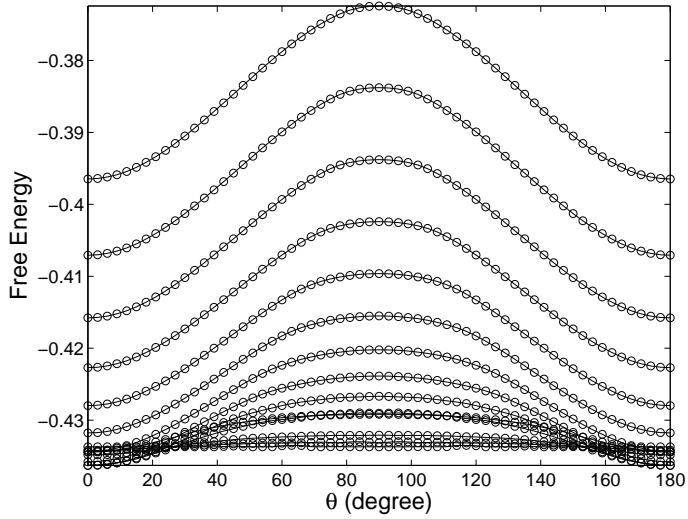

(a)

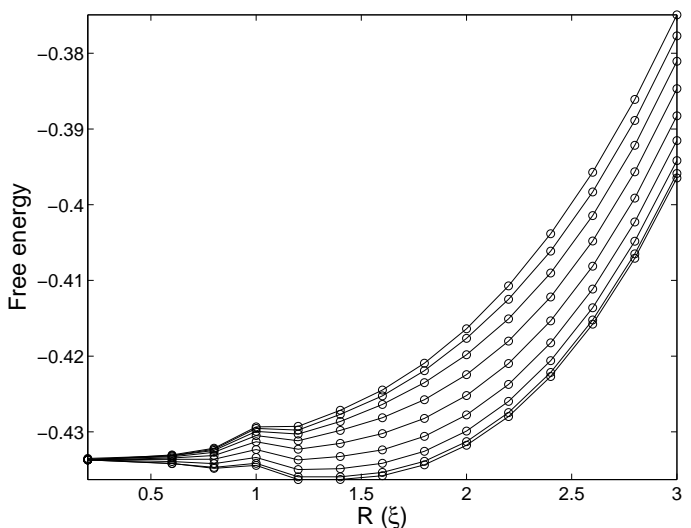

(b)

Figure 2. Dependence of the free energy, $\mathcal{F}_{c}$, with the pinning center radius $R$ and the angle $\theta$.

Fig. 2(a) - Variation of $\mathcal{F}_{c}$ as a function of the angle $\theta$ in the range $0^{\circ}$ to $180^{\circ}$, data points obtained for increments of $3^{\circ}$. The radius $R$ varies from $0.2 \xi$ to $3.0 \xi$ and for each increment of $0.2 \xi$ results in a distinct curve, all plotted in ascendant order from bottom to top.

Fig.2(b) - Variation of $\mathcal{F}_{c}$ as a function of the pinning center radius for a specific value of $\theta$. The radius varies from $0.2 \xi$ to $3.0 \xi$ with an increment of $0.2 \xi$. Distinct curve correspond to different $\theta$, equal to $0^{\circ}, 9^{\circ}, 18^{\circ}, 27^{\circ}, 36^{\circ}, 45^{\circ}, 54^{\circ}, 63^{\circ}$ and $72^{\circ}$ in the ascendant order form bottom to top.

The most significant advantage of the present method, is that the free energy functional, Eq. 3, contains the appropriate boundaries conditions to the problem. This removes the necessity of solving the theory in two independent regions and later applying the Neumman boundary conditions. Besides the present method easily applies to internal regions of any shape, not just spherical, and finds its solution for the given normal-superconductor interface.

\section{Results}

For a given pinning arrangement, which means fixed values of $\theta$ and $R$, and the condition of one vortex in the unit cell, we carry the minimization of the free energy. Here we present the results of several independent simulations obtained for the pairs $(\theta, R)$, ranging from $\left(0^{\circ}, 0.2 \xi\right)$ to $\left(180^{\circ}, 3.0 \xi\right)$, in increments of $3^{\circ}$ for $\theta$, and of $0.2 \xi$ for $R$.
For each simulation we initialize the order parameter in a random way and the minimization procedure is carried for each temperature ${ }^{1}$. Then the temperature is lowered until a convergence criteria is reached, which means that the free energy has become stable. For each simulation we implement at least 1200 Monte Carlo visits per mesh point in a Metropolis algorithm.

Our main results are shown in the two main curves of Fig. 3. The Fig. 2a shows several curves of the free energy density versus the angle $\theta$, each curve associated to a different $R$ value. Similarly the Fig. $2 \mathrm{~b}$ shows several free energy density versus $R$ curves, each corresponding to a distinct value of $\theta$. The Fig. 2a shows that mirror symmetry $\theta \leftrightarrow \pi-\theta$ holds as expected, since the two cavities inside the unit cell are equivalent. The free energy $\mathcal{F}_{c}(\theta, R)$ is an even function in $\theta$.

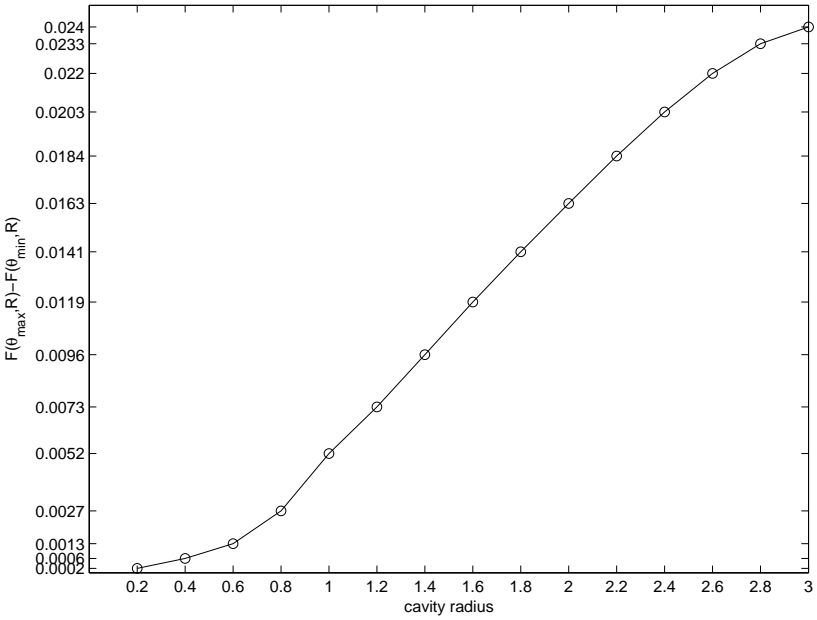

Figure 3. Amplitude of oscillation. $\mathcal{F}_{c}\left(90^{\circ}, R\right)-\mathcal{F}_{c}\left(0^{\circ}, R\right)$.

In our previous work $[16,17]$ we have found the remarkable property that cavities inside a superconductor can lower its energy as compared to the cavity-free superconductor. This effect can be verified here in Fig. 2b, which display a set of points lying under the energy threshold of -0.434 , the free energy $\mathcal{F}_{c}(\theta, R=0)$, of the system without cavities, approximated by $\mathcal{F}_{c}(\theta=0, R=0.2)$, as previously discussed. In summary we found here several pinning configurations, each described by the pair of values $(\theta, R)$, that have lower energy than the cavity free system. Table 1 exhibits these pairs $(\theta, R)$ of lower energy.

The curve of Fig. 2b shows monotonic growth [17] for the free energy, but only for $R$ equal or larger than $1.2 \xi$. It also shows a local maximum for the free energy at $R=$ $1.0 \xi$. In fact the behavior is distinct for the two regimes, $R>1.0 \xi$ and $R \leq 1.0 \xi$. To understand the $R>1.0 \xi$ case lut us hold the angle fixed, and vary the cavity radius. One finds that the free energy density is given by a constand density times the volume of the unit cell, $V_{0}=L^{3}$, removed of the

\footnotetext{
${ }^{1}$ The temperature here is the parameter associated with the metropolis algorithm. Its meaning is not related with the thermodynamical variable of the system.
} 
TABLE 1. Range of $\theta$ and $R$ values that establish a configuration with lower energy than the cavity-free superconductor.

\begin{tabular}{||c|c|c|c|c|c|c|c|c||}
\hline$\theta$ (degree) & $0-3$ & $0-15$ & $0-24$ & $0-12$ & $0-27$ & $0-24$ & $0-21$ & $0-9$ \\
\hline$R(\xi)$ & 0.4 & 0.6 & 0.8 & 1.0 & 1.2 & 1.4 & 1.6 & 1.8 \\
\hline
\end{tabular}

non-superconductor volume of the cavities, $V_{c}=8 / 3 \pi R^{3}$. This result is only approximately valid since the curvature of the order parameter near the pinning sphere surface causes an increase in the kinetic energy, an effect that becomes more pronounced for large spheres. Thus we have that for $R>1.0 \xi$

$$
\mathcal{F}_{c}\left(\theta_{\text {fixed }}, R\right) \propto \mathcal{F}_{c f}\left(1-\frac{8 \pi R^{3}}{3 L^{3}}\right),
$$

where $\mathcal{F}_{c f} \cong \mathcal{F}_{c}\left(0^{\circ}, 0.2 \xi\right)=-0.434$ is the cavity-free energy. The monotonic growth seen in Fig. $2 b$ is well described by the cubic $R$ dependence of Eq. 5 .

The dependence with the angle $\theta$ exhibited in Fig. 2a may be described by the expression

$$
\mathcal{F}_{c}\left(\theta, R_{\text {fixed }}\right) \propto f_{k}\left(R_{\text {fixed }}\right) \sin ^{2} \theta
$$

where the function $f_{k}(R)$ incorporate all the kinetics effects produced by the presence of the cavities with radius $R$. In Fig. 2a, $f_{k}(R)$ provides the amplitude of oscillation for a fixed $R$. As we have mentioned before, the kinetic effects become more pronounceable as the cavity radius increase. We assume that $f_{k}$ is a linear function of $R$.

We add Equations 5 and 6 together to obtain a general free energy expression to describe arbitrary angles of rotation and radii bigger than the coherence length:

$$
\mathcal{F}_{c}(\theta, R)=\mathcal{F}_{c f}\left(1-\frac{V_{c}}{V_{0}}\right)+f_{k}(R) \sin ^{2} \theta .
$$

For all radii, the configurations of minimum and maximum energies are obtained for $0^{\circ}$ and $90^{\circ}$, respectively. The maximum at $\theta=90^{\circ}$ has a smaller superconducting volume as compared to the minimum at $\theta=0^{\circ}$ configuration. This is easy to understand because the $0^{\circ}$ configuration has the two spheres aligned along the $\mathrm{z}$-axis and both overlap with the vortex line, whereas the $90^{\circ}$ configuration only one overlaps the vortex line. The other one is free in space thus taking away space that could be otherwise superconducting. This makes the $90^{\circ}$ configuration closer to the normal state than the $0^{\circ}$ one. At some intermediate angle between $0^{\circ}$ and $90^{\circ}$ a depinning transition takes place, although it is not noticeable in both Figs. $2 \mathrm{a}$ and $2 \mathrm{~b}$. This transition has been studied in Ref. [16]. The function $f_{k}(R)$ is easily obtained by taking its difference at extreme angles, $\mathcal{F}_{c}\left(\theta_{\max }, R\right)-\mathcal{F}_{c}\left(\theta_{\min }, R\right)$, where $\theta_{\max }=90^{\circ}$ and $\theta_{\min }=0^{\circ}$. This difference is shown in Fig. 3 as a function of $R$. Thus Eq. 7, the major conclusion of this paper, gives a good description of the free energy for all $(\theta, R)$ pairs.

In the limit that $R \rightarrow 0$, the free energy should converge to the cavity free superconductor, $\mathcal{F}_{c f}$, whose energy only depends on the unit cell vorticity. Thus the term $f_{k}$ should vanish in this limit $R \rightarrow 0$ so that the angular dependence $\sin ^{2} \theta$ disappears from the free energy:

$$
\lim _{R \rightarrow 0} f_{k}(R) \quad \rightarrow 0
$$

We determine the $f_{k}(R)$ term fitting the curve of the Fig. 3 with the best linear function. In this way, the function found is:

$$
f_{k}(R)=-0.00358+0.00962 R .
$$

The linear dependence of the Eq. 9 with the radius $R$ reflects the importance of the kinetic energy, $\xi^{2}\left|\left(\vec{\nabla}-\frac{2 \pi i}{\Phi_{0}} \vec{A}\right) \Delta\right|^{2}$, which describes the bending of the order parameter at the surface of the cavities, that becomes more important for large cavities.

Substituting all the terms in Eq. 7 by the terms obtained in the fitting and the parameters of the system, the energy dependence is expressed by following equation:

$$
\begin{aligned}
\mathcal{F}_{c}(\theta, R) & =\mathcal{F}_{c f}\left(1-\frac{8 \pi R^{3}}{3 L^{3}}\right) \\
& +(0.00962 R-0.00358) \sin ^{2} \theta
\end{aligned}
$$

The negative constant -0.00358 is in conflict with the condition expressed by Eq. 8. In fact in the region $R \leq 1.0 \xi$, Eq. 9 does not apply because of mesh effects. Besides for such small $R$ the present Ginzburg-Landau approach is not applicable, as previously discussed.

\section{Many vortices near the zigzag of cavities}

So far we have described the case of just one vortex near a zigzag of cavities, $\vec{\nu}=1 \hat{z}$. In this section we briefly comment on the general case of many vortices along the $z$ direction, $\vec{\nu}=n_{z} \hat{z}$. As $n_{z}$ increases the upper critical field is approached and beyond the upper critical field there is also a surface superconductivity state at the surface of the cavities. The radius of the cavities $R$ and the angle of rotation $\theta$ sets new geometric configurations in the unit cell that lead to multiple trapping and giant vortex states in the superconductor [13]. A simple example of such configurations is shown in Fig. 4, which displays two vortices in the unit cell $\left(n_{z}=2\right.$ case) for $R=1.8 \xi$ at the two extreme angles, $\theta=0^{\circ}$, and $\theta=90^{\circ}$. For $\theta=0^{\circ}$ the system presents strong competition between the vortex-vortex repulsion and the vortex-cavity attraction. While one of the vortices is barely trapped by the cavities the other vortex is not pinned. The rotation of the cavities to the $\theta=90^{\circ}$ configuration yields a state of two vortices each one trapped by a distinct cavity. For values of $n_{z}$ bigger than 2 more complex situations are possible and will be studied elsewhere. 


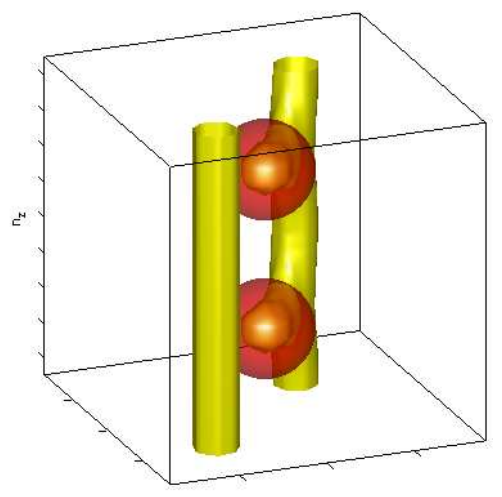

$n_{p}$

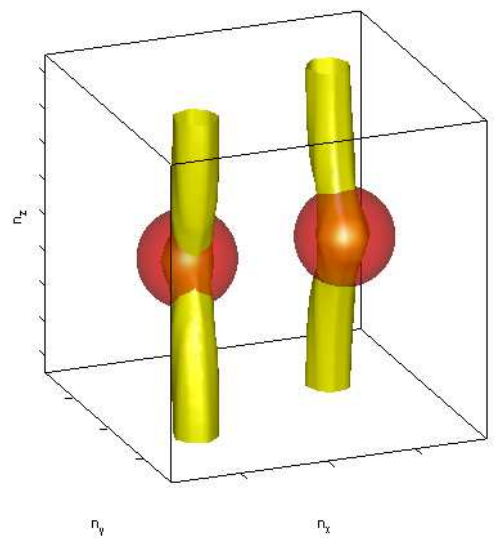

(b)

Figure 4. Iso-surfaces of the order parameter $|\Delta(\vec{x})|^{2}$ inside the unit cell, a cube with side $L=12 \xi$ containing cavities (in red) with radius $R=1.8 \xi$. The Fig. 4 a corresponds to $\theta=0^{\circ}$ and shows just one vortex line trapped by the defect, whereas the other one is not pinned and repelled due to vortex-vortex repulsion. In the Fig. $4 \mathrm{~b}$ the cavities form an angle $\theta=90^{\circ}$ and each vortex is trapped by an independent cavity.

\section{Conclusions}

We have carried here a Ginzburg-Landau theory study of a vortex line near a zigzag of pinning centers. The pinning centers are insulating spherical cavities and their arrangement is well described by a unit cell containing two of them. Inside the unit cell the rotation of the two cavities around the center produces a continuous of zigzag arrangements whose effects on the vortex line are here discussed. We observe distinct behavior for cavity radius above and below $1.0 \xi$. Sweeping the angle of rotation of the cavities around its center, $\theta$, makes the zigzag more pronounced to the point that the vortex line decouples from it above the critical angle $\theta_{c}$, as previously found [13]. Below this angle the vortex line is pinned by both cavities and above by just one. Here we have determined that the free energy density has a simple analytical dependence, expressed by the Eq. 7 .

\section{Acknowledgments}

Research supported in part by Instituto do Milênio de Nano-Ciências, CNPq, and FAPERJ (Brazil).

\section{References}

[1] J.-Y. Lin, M. Gurvitch, S. Tolpygo, A. Bourdillon, S. Hou, and J. M. Phillips. Phys. Rev. B, 54, 12717 (1996).

[2] V. V. Moshchalkov, M. Baert, V. V. Metlusko, E. Rossell, M. J. V. Bael, K. Temst, and Y. Bruynserade. Phys. Rev. B 57, 3615 (1998).

[3] V. Yurchenko, P. Lahl, S. Bunte, M. Jirsa, and R. Wordenweber. Physica C 404, 426 (2004)

[4] A. Bezryadin and B. Pannetier. J. of Low Temp. Phys. 98, 251 (1995).

[5] W. V. Pogosov and V. V. Moshchalkov. Physica C 404, 285 (2004).

[6] A. I. Buzdin. Phys. Rev. B 47, 11416 (1993).

[7] J. B. Ketterson and S. N. Song. Superconductivity. Cambridge University Press, 1st. edition, 1999.

[8] A. I. Larkin. Sov. Phys.-JETP 31, 784 (1970).

[9] E. H. Brandt. Rep. Prog. Phys. 58, 1465 (1995).

[10] D. J. P. Jr. and H. A. Fertig. Phys. Rev. B 67, 054504 (2003).

[11] G. S. Mkrtchyan and V. V. Shmidt. Soviet Physics JETP, 34, 195 (1972).

[12] M. M. Doria, S. C. B. de Andrade, and E. Sardella. Physica C, 341-348, 1199 (2000).

[13] M. M. Doria and G. F. Zebende. Phys. Rev. B 66, 064519 (2002).

[14] P. G. de Gennes. Superconductivity in Metals and Alloys. Persus Book, 2nd. edition, 1989.

[15] A. A. Abrikosov. Soviet Physics JETP, 5, 1174 (1957).

[16] M. M. Doria and A. R. de C. Romaguera. Europhys. Lett. 67, 446 (2004).

[17] A. R. de C. Romaguera and M. M. Doria. Eur. Phys. J. B 42, 3 (2004).

[18] The temperature here is the parameter associated with the metropolis algorithm. Its meaning is not related with the thermodynamical variable of the system. 